\title{
Scaling of global momentum transport in Taylor-Couette and pipe flow
}

\author{
Bruno Eckhardt ${ }^{1}$, Siegfried Grossmann ${ }^{1}$, and Detlef Lohse ${ }^{2}$ \\ ${ }^{1}$ Fachbereich Physik, Philipps-Universität Marburg, Renthof 6, D-35032 Marburg, Germany \\ 2 Department of Applied Physics and J. M. Burgers Centre for Fluid Dynamics, University of \\ Twente, 7500 AE Enschede, Netherlands
}

(October 29, 2018)

\begin{abstract}
We interpret measurements of the Reynolds number dependence of the torque in Taylor-Couette flow by Lewis and Swinney [Phys. Rev. E 59, 5457 (1999)] and of the pressure drop in pipe flow by Smits and Zagarola, [Phys. Fluids 10, 1045 (1998)] within the scaling theory of Grossmann and Lohse [J. Fluid Mech. 407, 27 (2000)], developed in the context of thermal convection. The main idea is to split the energy dissipation into contributions from a boundary layer and the turbulent bulk. This ansatz can account for the observed scaling in both cases if it is assumed that the internal wind velocity $U_{w}$ introduced through the rotational or pressure forcing is related to the the external (imposed) velocity $U$, by $U_{w} / U \propto R e^{\xi}$ with $\xi=-0.051$ and $\xi=-0.041$ for the Taylor-Couette ( $U$ inner cylinder velocity) and pipe flow ( $U$ mean flow velocity) case, respectively. In contrast to the RayleighBénard case the scaling exponents cannot (yet) be derived from the dynamical equations.

Pacs: 47.27.-i
\end{abstract}

\section{INTRODUCTION}

The relation between global flow properties and driving forces is interesting from a fundamental point of view and for upscaling from laboratory experiments to applications. Examples are the change in mean flow through a pipe as a function of pressure drop, the dependence of the heat transport as a function of temperature difference (Rayleigh-Benard (RB) flow), and the increase in torque required to maintain a certain rotation speed in a Taylor-Couette (TC) system. In all three systems the effects of the boundary layers are of prime importance but the way in which they are dealt with differs. For pipe flow the boundary effects are usually discussed in terms of the Prandtl-van Karman theory [1] which assumes a logarithmic law for the profile. f For the connection between mean flow and pressure drop it predicts an implicit logarithmic relationship, the so called skin friction law [1], in reasonable agreement with the experimental data.

On the other hand, Rayleigh-Bénard (RB) convection has mainly been discussed in terms of algebraic relations: In the last decade the power law relation $N u \sim R a^{2 / 7}$ between the Nusselt number $N u$ and the Rayleigh number $R a$ was thought to be an appropriate

\footnotetext{
${ }^{1}$ Barenblatt and coworkers however also succeeded to describe the data in terms of power laws [2].
} 
description of the experimental data [3, 4]. Recently, it has turned out that the dependences are more involved [5 7]. On the theoretical side, the analysis by Grossmann and Lohse [8] of the different dominant dissipation mechanisms leads to a detailed phase diagram for Rayleigh-Bénard convection that is in good agreement with the latest [6, 7] and older experiments. In this theory the relations between $N u$ and $R a$ are again algebraic.

For the Taylor-Couette system with rotating inner cylinder and resting outer one a description of the relation between the dimensionless torque $G$ and the Reynolds number $R e$ both in terms of a skin friction law and a pure power law has been tried [9, 10]. The dimensionless torque $G=T / \rho \nu^{2} L$ and the Reynolds number $R e=\Omega a(b-a) / \nu$ are defined with $T$ the torque, $\rho$ the fluid density and $\nu$ its kinematic viscosity, $L$ the length of the cylinders, $\Omega$ the angular rotation rate, and $b$ and $a$ the radii of the outer and inner cylinder, respectively. Lewis and Swinney's analysis [9] of their experimental data clearly shows that a pure power law $G \sim R e^{\alpha}$ with $\alpha=5 / 3$ as suggested in Refs. [11, 12] does not describe the data. A description in terms of the skin friction law is in better agreement with the data. However, it still is not fully satisfactory, either, as the systematic drifts in their Fig. 1 show.

It is our aim here to adopt Grossmann and Lohse's Rayleigh-Bénard theory to TaylorCouette and pipe flow. In contrast to the RB case, it is presently not possible to derive the scaling exponents fully from dynamical equations. Instead, there will be one exponent that has to be and can be fitted consistently to data for both systems.

We begin in section 2 with a discussion of the theory and the comparison to Lewis and Swinney's [9] experimental data for TC flow. In section 3 we will adopt it to pipe flow for which precise high $R e$ data on the pressure drop were obtained by Smits's group [13]. In section 4 we compare the quality of the data fit of this theory with that of the standard skin friction law theory. Section 5 gives conclusions.

\section{TAYLOR-COUETTE FLOW}

The basic idea [8] behind the analysis of the thermal convection experiments is the splitting of the energy dissipation $\epsilon$ into contributions from the boundary layer (BL) and the bulk,

$$
\epsilon=\epsilon_{B L}+\epsilon_{b u l k}
$$

For the energy dissipation $\epsilon$ in TC flow one strictly has 9

$$
\epsilon=\frac{\nu^{2} G \Omega}{2 \pi\left(b^{2}-a^{2}\right)}
$$

This can be derived as usual by considering the energy balance in a Navier-Stokes flow. In analogy to ref. [8] (see there for an extensive discussion) $\epsilon_{b u l k}$ is estimated as

$$
\epsilon_{b u l k} \sim \frac{U_{w}^{3}}{b-a}
$$

Here, $U_{w}$ is the typical velocity difference between the turbulent and the laminar (linear)

profile. It is a measure for the turbulent activity induced by the rotation of the inner cylinder and it defines a Reynolds number $R e_{w}=U_{w}(b-a) / \nu$. In the laminar case $U_{w}=R e_{w}=0$, 

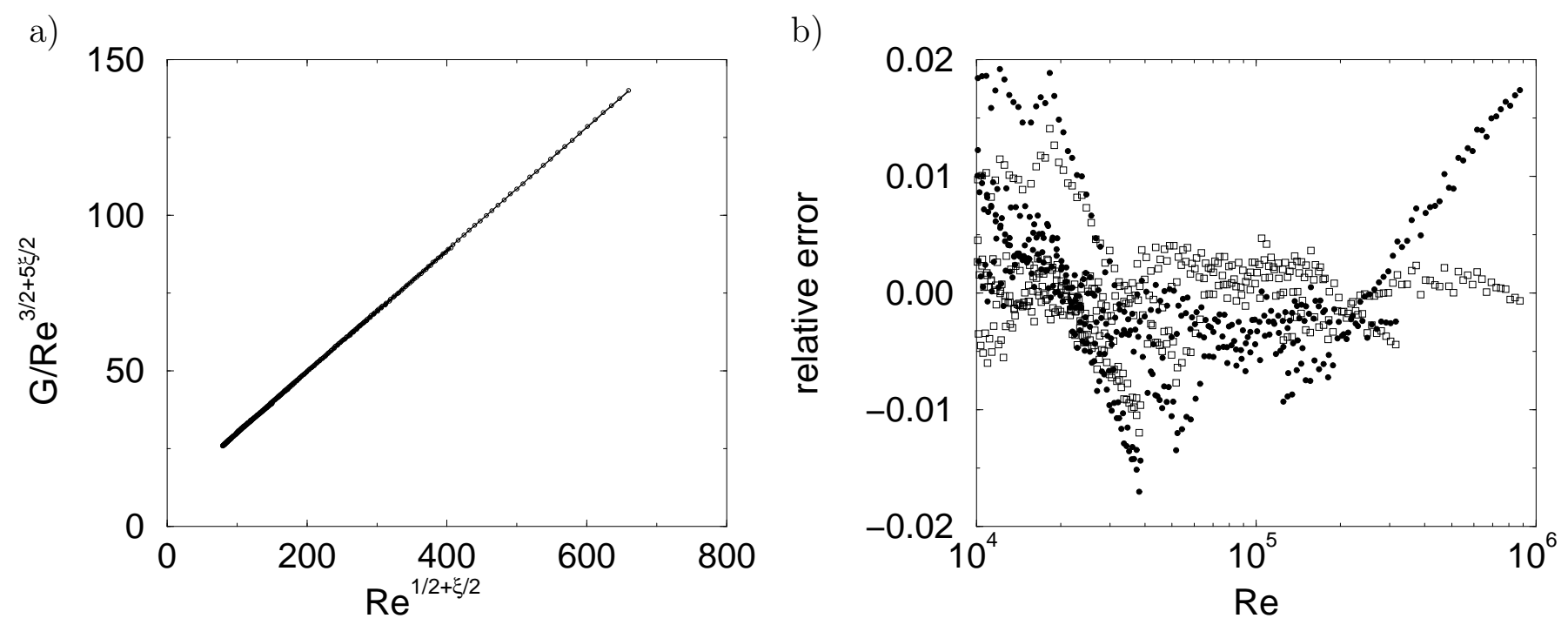

FIG. 1. a) Compensated plot $G / R e^{3 / 2+5 \xi / 2}$ vs $R e^{1 / 2+\xi / 2}$ with $\xi=-0.051$. The points are Lewis and Swinney's data [9], the line the fit (8). Re varies in the range $10^{4}$ through $9 \cdot 10^{5}$.

b) Relative error $\left(G-G_{f i t}\right) / G$ of the suggested combination of power laws (8) with $\xi=-0.051$ (open boxes) and relative error $\left(f-f_{f i t}\right) / f$ of the friction law fit (11) (filled circles).

and therefore $\epsilon_{b u l k}=0$. Obviously, $U_{w}$ must not be confused with the velocity $U=2 \pi a \Omega$ of the inner cylinder or the corresponding Reynolds number $R e=\Omega a(b-a) / \nu$. The Reynolds number $R e$ (or $U$ ) is imposed on the flow whereas $R e_{w}$ (or $U_{w}$ ) is the response of the system. The situation can be compared with $\mathrm{RB}$ convection where the Rayleigh number is imposed on the cell whereas the response of the system is the large scale wind of turbulence, which again defines a wind Reynolds number $R e_{w}$. Therefore, in analogy, also here we call $U_{w}$ the wind velocity.

It is this wind velocity which leads to the formation of a boundary layer of thickness $\lambda_{u}$. As in ref. [8] we assume the BL to be of Blasius type [1],

$$
\lambda_{u} \sim(b-a) / \sqrt{R e_{w}} .
$$

For very small $R e_{w}$ the BL will of course not diverge but saturate at a scale $\lambda_{u} \sim(b-a)$ which introduces different scaling relations [14], but in the present work we are not interested in this very low Reynolds number regime.

With its thickness $\lambda_{u}$ as the relevant length scale we estimate the energy dissipation in the BL as 8

$$
\epsilon_{B L} \sim \nu \frac{U_{w}^{2}}{\lambda_{u}^{2}} \frac{\lambda_{u}}{b-a} .
$$

Putting eqs. (2) to (5) together one obtains

$$
G R e=c_{1} R e_{w}^{5 / 2}+c_{2} R e_{w}^{3}
$$


where $c_{1}$ and $c_{2}$ are two unknown constants. The first term is the BL contribution, the second one the bulk contribution.

The central question now is: How does $R e_{w}$ depend on $R e$ ? We do not know, but for large enough $R e$ it seems reasonable to assume a power law dependence,

$$
\frac{R e_{w}}{R e} \sim \frac{U_{w}}{U} \sim R e^{\xi}
$$

Therefore,

$$
G=c_{1} R e^{3 / 2+5 \xi / 2}+c_{2} R e^{2+3 \xi}
$$

We perform a nonlinear fit of Lewis and Swinney's data 99 to eq. (8), obtaining $\xi=-0.051$, $c_{1}=10.5$, and $c_{2}=0.196$. The best way to check the quality of the fit (8) is to plot $G / R e^{3 / 2+5 \xi / 2}$ vs $R e^{1 / 2+\xi / 2}$ so that according to eq. (8) a straight line should result. This indeed is the case, as shown in Fig. 1a. The quality of the fit is underlined by the relative error shown in Fig. 1p.
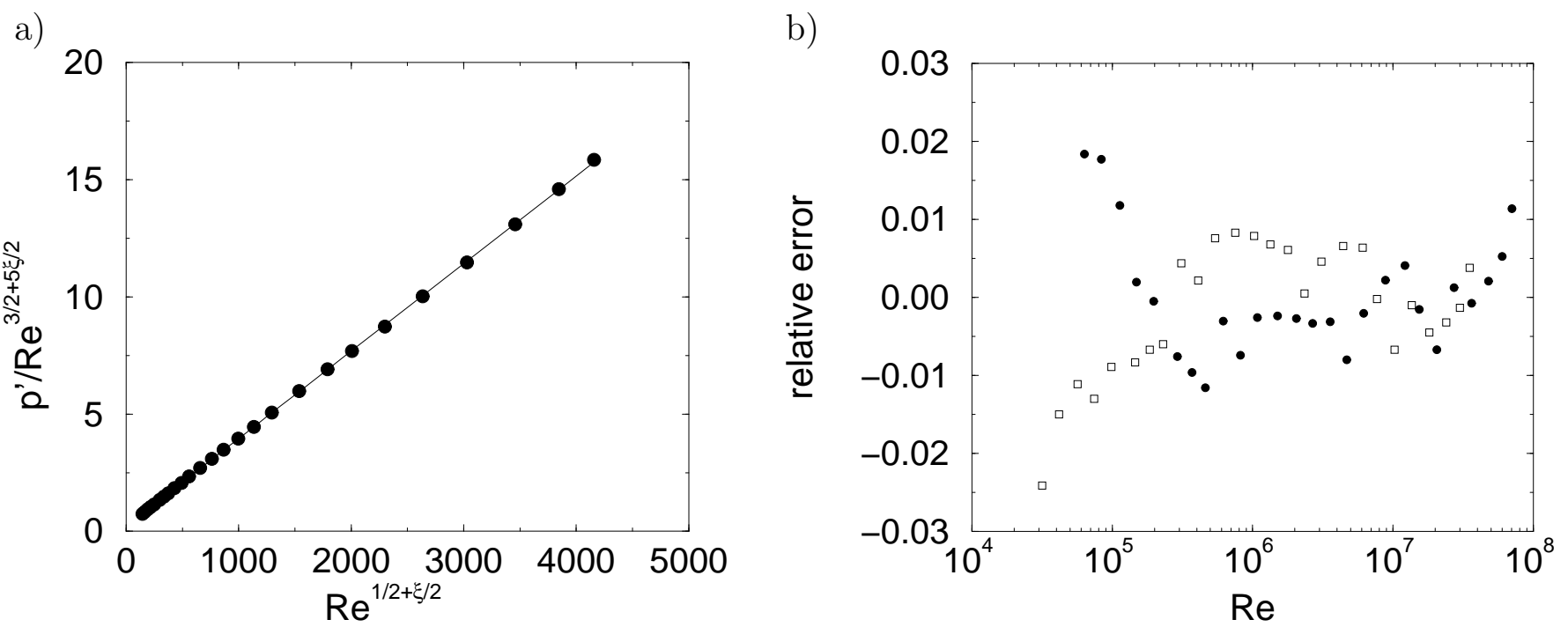

FIG. 2. a) Compensated plot $p^{\prime} / R e^{3 / 2+5 \xi / 2}$ vs $R e^{1 / 2+\xi / 2}$ with $\xi=-0.041$. The points are data from ref. [13], the line the fit (10). Re here varies between $3.16 \cdot 10^{4}$ and $3.53 \cdot 10^{7}$.

b) Relative error $\left(p^{\prime}-p_{f i t}^{\prime}\right) / p^{\prime}$ of the suggested combination of power laws (10) with $\xi=-0.041$ (open boxes) and relative error $\left(f-f_{f i t}\right) / f$ of the friction law fit (11) (filled circles).

\section{PIPE FLOW}

Consider now pressure driven flow through a pipe of radius $R$. The pressure gradient and the energy dissipation $\epsilon$ per volume are related by

$$
\epsilon=\frac{\Delta p}{\rho} \overline{u_{x}}
$$


where $\overline{u_{x}}=: U$ is the average of the $x$-velocity over the cross section of the pipe, which defines the Reynolds number $R e=2 R U / \nu$. As for the TC flow we define the wind velocity $U_{w}$ as the maximal difference between the turbulent mean velocity profile and the laminar (parabolic) one. This maximum will occur close to the walls. $U_{w}$ again defines a wind Reynolds number $R e_{w}=2 R U_{w} / \nu$.

We split the energy dissipation in a boundary layer part and a bulk part as in equation (10) and estimate both contributions as above, eqs. (3) and (5), with $b-a$ replaced by $R$, and the thickness of the Blasius boundary layer being $\lambda_{u} \sim R / \sqrt{R e_{w}}$. With this the equation for the dimensionless pressure drop $p^{\prime}=\Delta p R^{3} /\left(\rho \nu^{2}\right)$ becomes

$$
p^{\prime}=c_{1}^{\prime} R e^{3 / 2+5 \xi / 2}+c_{2}^{\prime} R e^{2+3 \xi},
$$

where $c_{1}^{\prime}$ and $c_{2}^{\prime}$ are two unknown constants and $\xi$ the power law exponent of eq. (7). Equation (10) is the analog of equation (8) in the TC case.

In order to test equation (10) we consider the high precision pressure drop data of Smits and Zagarola [13]. A nonlinear fit then results in $\xi=-0.041$ and $c_{1}^{\prime}=0.226$ and $c_{2}^{\prime}=0.00373$. In Fig. 2a we show $p^{\prime} / R e^{3 / 2+5 \xi / 2}$ vs $R e^{1 / 2+\xi / 2}$. If eq. (10) holds, a straight line should result which is the case. Again the quality of the fit is demonstrated with the relative errors in Fig. 2 $\mathrm{b}$.

\section{THE SKIN FRICTION LAW}

Finally we would like to compare our description of the data with the standard skin friction law [1]. Define the friction coefficient $f=G / R e^{2}$ for TC flow and $f=p^{\prime} / R e^{2}$ for pipe flow. One then has, in both cases [1, 9, 10],

$$
\frac{1}{\sqrt{f}}=c_{1}^{\prime \prime} \lg (\operatorname{Re} \sqrt{f})+c_{2}^{\prime \prime}
$$

with two flow dependent constants $c_{1}^{\prime \prime}$ and $c_{2}^{\prime \prime}$ which can be connected to the von Karman constant [9]. Employing equation (11) one can indeed fit both data sets reasonably. We do not show the fits as they have already been shown elsewhere (see figure 4a of ref. [9] for the TC case), but we present the relative error $\left(f-f_{f i t}\right) / f$ in figures 1 1a and 2 $2 \mathrm{~b}$ and compare it with the relative errors of the combined power laws eqs. (8) and (10), respectively. For both $\mathrm{TC}$ and pipe flow the relative error of the friciton law is roughly of the same order as that of the fits (8) and (10), respectively, maybe somewhat larger.

\section{CONCLUSIONS}

The preceeding analysis shows that the splitting of the dissipation into a bulk and a boundary layer contribution as used in the Rayleigh-Bénard theory can also be used to describe the Taylor-Couette flow [9] and the pipe flow [13] data. However, in contrast to the $\mathrm{RB}$ case, only one global balance equation is available, the one for the energy dissipation. Therefore, it is not possible to derive the asymptotic scaling exponents for both the wind Reynolds number and the dimensionless torque (dimensionless pressure drop) in the TC 
case (pipe case). Instead, one scaling exponent $(\xi)$ must be fitted to the data. The ratio $U_{w} / U \sim R e^{\xi}$ scales similarly in both cases, $\xi=-0.051$ and $\xi=-0.041$ for TC and pipe flow, respectively. The relative error in both cases is less than one percent and within this precession the data indicate really different exponents. The origin of this difference is unclear.

For shear flow, the strict upper bound for energy dissipation is $c_{\epsilon}=\epsilon L / U^{3} \leq 0.01087$ for $R e \rightarrow \infty$ [15, 16]. All the Couette experiments clearly lie below this bound, and even show a trend towards a scaling that is slower than $U^{3}$. When assuming the Kolmogorov length scale as smallest length scale on which dissipation contributes, Nicodemus et al. [17] could numerically show that around $R e=10^{5}-10^{6}$ one has $c_{\epsilon} \sim R e^{-0.08}$. Kerswell assumes the same cutoff and a certain background flow profile and finds $c_{\epsilon} \sim R e^{-1 / 7}$ for $R e \rightarrow \infty$ [18]. Interestingly enough, the exponents $3 \xi=-0.12$ (TC) and $3 \xi=-0.15$ (pipe) that we find from the experimental data are very close to that value. However, the scaling $U_{w} / U \sim R e^{\xi}$ with negative $\xi$ implies that the wind does not increase as rapidly as the external velocity with $R e$ and that according to our definition of $U_{w}$ the relative difference between the laminar and the mean turbulent velocity profiles vanishes. Given the smallness of $\xi$ the Reynolds numbers at which this could become significant are not experimentally accessible. But the situation remains unsatisfactory and it clearly would be highly desirable to calculate this exponent more rigorously from the Navier-Stokes equations and to understand the relation to the mean flow profile better.

Acknowledgements: The authors thank Ch. Doering and K. R. Sreenivasan for very helpful discussions and H. Swinney, G. Lewis, A. J. Smits, and M. V. Zagarola for supplying us with their experimental data. The work is part of the research program of the Stichting voor Fundamenteel Onderzoek der Materie (FOM), which is financially supported by the Nederlandse Organisatie voor Wetenschappelijk Onderzoek (NWO). This research was also supported by the German-Israeli Foundation (GIF), by the European Union (EU) under contract HPRN-CT-2000-00162, and in part by the National Science Foundation (NSF) under Grant No. PHY94-07194. We thank the members of the Institute for Theoretical Physics in Santa Barabara for their hospitality.

[1] L. D. Landau and E. M. Lifshitz, Fluid Mechanics (Pergamon Press, Oxford, 1987).

[2] G.J. Barenblatt, J. Fluid Mech. 248, 513 (1993);

G.J. Barenblatt, A. Chorin, and V.M. Prostokishin, Appl. Mech. Rev. 50, 413 (1997).

[3] B. Castaing et al., J. Fluid Mech. 204, 1 (1989).

[4] E. D. Siggia, Annu. Rev. Fluid Mech. 26, 137 (1994).

[5] X. Chavanne et al., Phys. Rev. Lett. 79, 3648 (1997).

[6] J. Niemela, L. Skrebek, K. R. Sreenivasan, and R. Donelly, Nature 404, 837 (2000).

[7] X. Xu, K. M. S. Bajaj, and G. Ahlers, Phys. Rev. Lett. 84, 4357 (2000).

[8] S. Grossmann and D. Lohse, J. Fluid. Mech. 407, 27 (2000).

[9] G. S. Lewis and H. L. Swinney, Phys. Rev. E 59, 5457 (1999). 
[10] D. P. Lathrop, J. Fineberg, and H. S. Swinney, Phys. Rev. A 46, 6390 (1992).

[11] A. Barcilon and J. Brindley, J. Fluid Mech. 143, 429 (1984).

[12] G. P. King, Y. Li, H. L. Swinney, and P. S. Marcus, J. Fluid. Mech 141, 365 (1984).

[13] A. J. Smits and M. V. Zagarola, Phys. Fluids 10, 1045 (1998).

[14] S. Grossmann and D. Lohse, Preprint (2000).

[15] F. H. Busse, Adv. Appl. Mech. 18, 77 (1978).

[16] R. Nicodemus, S. Grossmann, and M. Holthaus, Phys. Rev. Lett. 79, 4170 (1997); Phys. Rev. E 56, 6774 (1997); J. Fluid Mech. 363, 281 (1998); J. Fluid Mech. 363, 301 (1998).

[17] R. Nicodemus, S. Grossmann, and M. Holthaus, Eur. Phys. J. B 10, 385 (1999).

[18] R. R. Kerswell, Preprint (2000). 